57.9 (SD 5.32) and a mean disease duration of 13.36 years (SD 9.25).

Results Six months after training, participants reported small but significant increases in arthritis self-efficacy pain $(\mathrm{p}=0.002)$, cognitive symptom management $(p=0.004)$ and communication with health practitioners $(\mathrm{p}=0.024)$, and a small but significant decrease in depression $(\mathrm{p}=0.04)$. Qualitative data supported these findings with participants reporting more confidence, happiness and a changed outlook on life in general.

Conclusions Improvements reported by study participants lend support to the value of volunteerism and training to become lay leaders on an arthritis self-management programme. Volunteers noticed positive changes both in themselves and course participants. Many had begun to apply their new found knowledge about self-management to their own situation, reporting less pain and more willingness 'to get on with life'.

\section{SP0162 A LONGITUDINAL STUDY OF THE NEED FOR CONCORDANCE}

BA Chewning, J Wiederholt. Pharmacy, University of Wisconsin, Madison, USA

\subsection{6/annrheumdis-2001.1252}

Background In the past 5 years, there has been growing acknowledgment that patients need to be active in their medication management. The Client Centred Model (1) as well as the Concordance Framework (2) stress the importance of providers supporting patients to assume an active role in monitoring and evaluating their regimen benefits and side effects. Particularly as prescription drug use continues to grow (37\% increase in the number of prescriptions dispensed between 1992-1998 in the US (3)), there is an urgent need to conceptualise and reframe how patient-provider partnerships apply specifically to medication management.

Objectives This paper presents findings regarding how people with arthritis attempt to control their ever changing, complex regimens over time. Second, this paper examines the potential of the Concordance Framework and Client-Centred Model for reframing the nature of the patient-provider partnership to enhance patients' sense of control related to their overall quality of life. We will explore how the concept of Concordance alters our view of the frequently complex and frustrating process of medication management.

Method A longitudinal study of 689 patients with the formal diagnosis of osteoarthritis (OA) or rheumatoid arthritis (RA) was conducted to identify patient perceptions, decision-making and behaviour related to their medication management. Face to face interviews with the Brief Medication Questionnaire (BMQ), health quality of life questionnaires (AIMS2 and SF-36) and telephone interviews were conducted in waves centred around patient visits every 6 months for 2 years. Data about medication regimens were abstracted from the clinic medication profile record for each visit.

Setting Three rheumatology clinics in the United States participated in the study: 1) a university clinic; 2) a private clinic; 3) a Veterans Administration clinic. Patients were enrolled at the time of a regular clinic visit.

Key findings At baseline, 50\% of patients had at least one comorbidity and $22 \%$ had at least two comorbidities. Almost half $(48 \%)$ of patients in the sample had complex regimens with 8 or more medications at any one time period. The majority of physicians altered their medication orders every 6 months for patients. Hence many patients faced complex, shifting regimens. Patients reported they evaluated the effectiveness and side effects of individual medicines based largely on symptoms. There were 248 reported deviations in the scheduled medications at baseline and the majority (61\%) were intentional, largely based on symptoms.

These deviations are viewed differently when one applies the compliance perspective versus the Concordance Framework. While the compliance perspective is somewhat pejorative with respect to patient behaviour, Concordance suggests the need for greater agreement between patient and health professional regarding how best to calibrate and manage the regimen to meet the patient's priorities. The longitudinal nature of our study shifted attention away from compliance to the process of seeking better regimens in the absence of "perfect regimens". Implications for the psychology of managing arthritis regimens between visits and the need for patient-provider partnerships using both the unique expertise of the patient and provider are explored.

\section{Rehabilitation - physio/occupational therapy}

\section{HP0024 APPLICABILITY OF AN EDUCATIONAL-BEHAVIOURAL JOINT PROTECTION PROGRAMME IN PRACTICE}

${ }^{1}$ A Hammond, ${ }^{2} \mathrm{P}$ Jeffreson, ${ }^{2} \mathrm{~N}$ Stacey. ${ }^{1}$ Rheumatology, Derbyshire Royal Infirmary, Derby; ${ }^{2}$ Occupational Therapy, Robert Jones and Agnes Hunt Orthopaedic Hospital, Oswestry, UK

\subsection{6/annrheumdis-2001.1253}

Background Previous clinical trials proved an educational-behavioural joint protection (EB-JP) programme is more effective than "standard" teaching in increasing people with rheumatoid arthritis' (RA) use of joint protection (JP).

Objective This study aimed to investigate if rheumatology occupational therapists (OT), following a 2 day training course, can in clinical practice similarly enable people with RA to increase use of JP.

Methods The study was conducted with 3 trained OTs at 3 hospitals using a randomised, crossover design. Demographic, Patient Knowledge Questionnaire (PKQ), hand and functional status measures were recorded at baseline, 3 and 6 months. The main outcome was the Joint Protection Behaviour Assessment (JPBA): a valid, reliable observational assessment of JP hand actions in kitchen activities.

Results 30 people with RA participated: 27 women and 3 men, average age 52.3 years (SD 12.1) with average disease duration of 6.76 years (SD 7.02). There was no significant difference in JPBA scores at baseline between the two groups: E1 (education first $)=17 \%($ SD 15\%) and E2 (education delayed $)=20 \%(S D$ $7.4 \%)(\mathrm{p}=0.16)$. At 3 months E1 JPBA scores significantly increased to $41 \%$ (SD 15.9\%) and E2 did not change: 25\% (SD $20 \%)(p=0.003)$. Both groups increased use of JP overall at 6 months $(\mathrm{p}<0.01)$. There was a significant increase in PKQ scores $(\mathrm{p}<0.00)$. No change in hand and functional status occurred, apart from increased grip strength $(p<0.01)$, but the follow-up period was probably too short to identify improvements. Most participants considered the programme relevant to their needs and found the emphasis on practical training very helpful.

Conclusion A 2 day training course, plus detailed programme manual, enables rheumatology OTs to deliver this programme successfully in practice, suggesting this programme should now be disseminated. 